\title{
Global hydro-climatic biomes identified via multitask learning
}

\author{
Christina Papagiannopoulou ${ }^{1}$, Diego G. Miralles ${ }^{2}$, Matthias Demuzere ${ }^{2}$, Niko E. C. Verhoest ${ }^{2}$, and \\ Willem Waegeman ${ }^{1}$ \\ ${ }^{1}$ Department of Data Analysis and Mathematical Modelling, Ghent University, Ghent, Belgium \\ ${ }^{2}$ Laboratory of Hydrology and Water Management, Ghent University, Ghent, Belgium
}

Correspondence: Christina Papagiannopoulou (christina.papagiannopoulou@ugent.be)

Received: 30 March 2018 - Discussion started: 25 April 2018

Revised: 21 August 2018 - Accepted: 29 August 2018 - Published: 12 October 2018

\begin{abstract}
The most widely used global land cover and climate classifications are based on vegetation characteristics and/or climatic conditions derived from observational data. However, these classification schemes do not directly stem from the characteristic interaction between the local climate and the biotic environment. In this work, we model the dynamic interplay between vegetation and local climate in order to delineate ecoregions that share a coherent response to hydro-climate variability. Our novel framework is based on a multitask learning approach that discovers the spatial relationships among different locations by learning a low-dimensional representation of predictive structures. This low-dimensional representation is combined with a clustering algorithm that yields a classification of biomes with coherent behaviour. Experimental results using global observation-based datasets indicate that, without the need to prescribe any land cover information, the identified regions of coherent climate-vegetation interactions agree well with the expectations derived from traditional global land cover maps. The resulting global "hydro-climatic biomes" can be used to analyse the anomalous behaviour of specific ecosystems in response to climate extremes and to benchmark climate-vegetation interactions in Earth system models.
\end{abstract}

\section{Introduction}

Approaches which aim to define regions with similar biophysical characteristics are commonly known as land cover classification schemes and are widely used in multiple geoscientific disciplines. Land cover classifications are crucial to enable a better understanding of the spatial variability of the land surface, which can be a first and necessary step towards understanding complex spatio-temporal interactions among different environmental variables (Feddema et al., 2005). Traditional land use and/or land cover (change) classifications are typically based on spectral information from the land surface coming from satellites (Loveland and Belward, 1997; Congalton et al., 2014). Amongst the most well known and widely used are the International GeosphereBiosphere Program DISCover Global $1 \mathrm{~km}$ Land Cover classification (IGBP-DIS) (Loveland et al., 2000), Global Land Cover 2000 (Bartholomé and Belward, 2005), and more recently the land cover map developed within the European Space Agency's Climate Change Initiative (ESA CCI) (Poulter et al., 2015; Li et al., 2018). Similarly, climate classification schemes cluster regions with similar climate conditions and are also widely used to stratify geographical regions with different climatic expectations (Baker et al., 2009; Brugger and Rubel, 2013; Garcia et al., 2014; Herrando-Pérez et al., 2014). Here, the best known is probably the Köppen-Geiger climate classification (Köppen, 1936), which has been modified many times in recent decades (e.g. Thornthwaite, 1943; Trewartha and Horn, 1980; Feddema, 2005; Kottek et al., 2006; Peel et al., 2007). Yet to date, dynamics in these climate regimes are used as a diagnostic of climate change by exploring their shifting boundaries (e.g. Diaz and Eischeid, 2007; Chen and Chen, 2013; Zhang and Yan, 2014a, b; Spinoni et al., 2015; Chan and Wu, 2015) or as a means to predict future climatic zone distributions using climate projections (e.g. Hanf et al., 2012, Gallardo et al., 2013, Mahlstein et al., 2013).

In recent years, the exponential advance in Earth observation research has made climate science one of the most data-rich scientific domains (Faghmous and Kumar, 2014). 
As such, data-driven methods have become popular in their use for land cover and climate classifications. For instance, Lund and Li (2009) proposed a new distance measure to define seasonal means and autocorrelations of climatic time series from weather stations and grouped the stations using a hierarchical agglomerative clustering. Zscheischler et al. (2012) also stressed the importance of unsupervised methods for tasks such as the classification of the land surface into zones with different climate and vegetation characteristics. Metzger et al. (2012) applied an alternative data-driven approach on climate and vegetation data that used principal component analysis (PCA) to discover informative structures in the data. In this method, the principal components of the initial climate-vegetation dataset were applied as input to a clustering algorithm. Interesting results in the same direction can be attributed to Netzel and Stepinski (2016, 2017), who used distance measures of climatic variables, such as dynamic time warping, coming from the time series analysis in a data mining approach. In addition, temporal change in climate zones has been explored in the same context via clustering algorithms, such as $k$ means (Zhang and Yan, 2014a, b). Finally, data-driven methods have been also applied for the biome classification task, which has been commonly treated as an object recognition problem using remote sensing data. In this case, techniques coming from computer vision are frequently applied (Mekhalfi et al., 2015; Chen and Tian, 2015). Following the progress in computer science, neural networks and deep learning approaches are also becoming popular for this kind of tasks in recent years, making the whole procedure even more automated (Scott et al., 2017; Xu et al., 2018).

Previous studies rely on spectral information, supervised techniques, or clustering approaches, which are applied to observations of climate variables and/or vegetation characteristics. However, these classification schemes are not based on the type of response of vegetation to climate dynamics. Recent advances in understanding vegetation response to climate variability highlight the importance of revealing the sensitivity of ecosystems to climate conditions; see Nemani et al. (2003), De Keersmaecker et al. (2015), Seddon et al. (2016), Papagiannopoulou et al. (2017b), or Liu et al. (2018). Therefore, a step beyond these previous studies is a spatial characterization of the vegetation dynamics that are induced by climate variability so that ecosystems of similar response to climate anomalies can be unveiled. This objective could be tackled by geostatistical approaches, such as geographically weighted regression (GWR) (Brunsdon et al., 1996), which assume that neighbouring pixels have a similar behaviour with respect to specific variables; these methods have already been applied in studies with a regional focus (Propastin et al., 2008; Zhao et al., 2015; Georganos et al., 2017). However, here, we aim to avoid neighbourhood assumptions and focus on the discovery of relationships between pixels based on the similarity in their modelled climate-vegetation interaction, acknowledging that global ecosystems may experience sim- ilar interactions even if they are remotely located from each other. A previous effort towards detecting regions with similar vegetation response to climate involves the work of Ivits et al. (2014), in which PCA is performed on the data matrix of drought anomalies and vegetation state, and a clustering is applied to the correlation coefficients based on the spatiotemporal patterns obtained by PCA. However, in this study, the interaction between climate and vegetation is not explicitly learned, nor are the causes behind vegetation changes inferred in a predictor-target framework.

Here, we introduce for the first time (to the best of our knowledge) a data-driven approach that aims to quantify the response of vegetation to local climate variables in a supervised setting at a global scale and use this information to define ecoregions of consistent behaviour against hydroclimatic variability. In simple terms, our framework results in regions where vegetation responds similarly to the dynamics in temperature, soil moisture, and incoming radiation. The proposed framework relies on predictive modelling and clustering techniques and builds further upon recent work in which we investigated the global response of vegetation to local climate by applying machine-learning algorithms in a Granger causality setting (Papagiannopoulou et al., 2017a, b). Since we aim to exploit the relationships between different pixels - instead of modelling each pixel separately as in our previous work - we propose the use of multitask learning (MTL) methods (Caruana, 1997). These methods are commonly used for solving multiple related tasks: considering as one task the prediction of vegetation in one location and as multiple tasks the prediction of vegetation in multiple locations, we can model our problem by using an MTL approach. First, we apply an MTL approach which tries to unveil lowdimensional common predictive structures and exploit the relationships among them. Second, we employ a clustering technique on these informative structures, which is applied on a lower dimensional space (Sect. 2). This clustering technique is known as spectral clustering ( $\mathrm{Ng}$ et al., 2001) and is one of the core assets of our framework. We refer to the emergent regions of coherent vegetation-climate behaviour as hydro-climatic biomes (Sect. 3).

\section{Methodology}

\subsection{Datasets}

We have built a large database of global climate and vegetation data that will be used in the context of our framework. These data are described in detail in Papagiannopoulou et al. (2017a) and are mostly based on satellite and/or in situ observations. The database spans a 30-year period (1981-2010) at monthly temporal resolution and $1^{\circ}$ latitudelongitude spatial resolution. The most important climatic and environmental drivers of vegetation are included, namely (i) land surface temperature, (ii) near-surface air temperature, 
(iii) longwave-shortwave surface radiative fluxes, (iv) precipitation, (v) snow water equivalent, and (vi) soil moisture. To characterize vegetation, we use the Global Inventory Modelling and Mapping Studies (GIMMS) NDVI3g dataset (Tucker et al., 2005). The target variable of our machinelearning framework is the de-trended seasonal NDVI anomalies. These are calculated through a simple linear de-trending and a multi-year average for each month of the year to capture the seasonal expectation - see Papagiannopoulou et al. (2017a) for more details. All other datasets describing the multi-month local climate variability over the 3 -decade period are used as predictor variables.

In addition, a wide range of "high-level features" have been handcrafted from the raw time series of predictors and used in addition to predictor variables. As such, our set of predictive features includes not just the raw data time series of each climate and/or environmental variable, but also seasonal anomalies, de-trended seasonal anomalies, lagged variables, past cumulative variables, and extreme indices - see Papagiannopoulou et al. (2017a). The cumulative variables capture the climatic conditions up to present time; an example would be the precipitation of the last (e.g.) 3 months. Extreme indices include maximum and minimum values, consecutive dry days, and values for specific percentiles. The use of these non-linear features (non-linear due to the way that they have been calculated) greatly improves causal inference and helps characterize non-linear relationships between climate and vegetation dynamics, as shown in our recent work (Papagiannopoulou et al., 2017a). For further discussion about the importance of this higher-level feature representation adopted in our framework, we refer the reader to Sect. S1 of the Supplement.

\subsection{Pixel-based approach: single-task learning}

In our study, we use information on climate and vegetation variables at specific time points and locations. Formally, we consider a spatio-temporal dataset $D=$ $\left\{\left(\mathbf{X}^{(1)}, \boldsymbol{y}^{(1)}\right),\left(\mathbf{X}^{(2)}, \boldsymbol{y}^{(2)}\right), \ldots,\left(\mathbf{X}^{(L)}, \boldsymbol{y}^{(L)}\right)\right\}$, with $L$ being the number of different locations and $\left(\mathbf{X}^{(l)}, \boldsymbol{y}^{(l)}\right)$ the tuple of the predictor variables and the target variable of each location $l$. We denote $D^{(l)}=\left\{\left(\boldsymbol{x}_{i}^{(l)}, y_{i}^{(l)}\right)\right\}_{i=1, \ldots, N}$ the observations of a location $l$, while the input feature vectors (i.e. the set of climatic variables) are denoted as a matrix $\mathbf{X}^{(l)}=\left[\boldsymbol{x}_{1}^{(l)}, \ldots, \boldsymbol{x}_{N}^{(l)}\right]^{T}$ and the corresponding target values as $\boldsymbol{y}^{(l)}=\left[y_{1}^{(l)}, \ldots, y_{N}^{(l)}\right]^{T}$ (i.e. the NDVI anomalies). Specifically, $\mathbf{X}^{(l)} \in \mathbb{R}^{N \times d}$ is the matrix of the predictor variables with $d$ being the number of predictors and $\boldsymbol{y}^{(l)} \in \mathbb{R}^{N}$ the response time series (i.e. NDVI seasonal de-trended anomalies), where $N$ denotes the number of discrete time stamps, i.e. the length of the time series. In this setting, a straightforward approach is to tackle each regression problem in each location $l$ separately, i.e. by independently training one model for each location (Papagiannopoulou et al., 2017a). That way, for every pixel only the data of that particular location $l$ are used $\left(\left(\mathbf{X}^{(l)}, \boldsymbol{y}^{(l)}\right), l=1, \ldots, L\right)$, not attempting to utilize the data from other regions where the target variable might have a similar response to the predictors.

We can start by defining regions of similar climatevegetation dynamics with the most naive approach: the relationship between climate and vegetation can be caught by the weights of a simple regression model, i.e. the regression coefficients of the predictor variables. Specifically, if one defines a simple linear regression model for a location $l$, the model for the $l$ th location is given by $f^{(l)}\left(\boldsymbol{x}_{i}^{(l)}\right)=\boldsymbol{w}^{(l)} \boldsymbol{x}_{i}^{(l)}$, with $\boldsymbol{x}_{i}^{(l)}$ being the input data (i.e. one observation) and $\boldsymbol{w}^{(l)}$ being the weight vector learned for particular location $l$, which describes the importance of each input variable for the target - see Fig. 1a. Even though one can assume that these weight vectors can be similar for regions in which the response of vegetation to climate is similar, the information from these other regions is not used in the prediction (i.e. each regression is applied at each individual pixel separately). This is despite the fact that these locations could be subsequently grouped (e.g. based on a similarity measure of their weight vectors) into wider regions that one may assume share common climate-vegetation dynamics. Note also that the information captured by each weight vector $\boldsymbol{w}^{(l)}$ should be sufficient, which means that it is necessary for the models to have a good generalization performance.

\subsection{Exploiting spatial relationships: multitask learning}

Unlike the single-task learning models described above that only take the data of each particular location into account, MTL models extract information of datasets with similar characteristics from other locations. As such, they can be expected to generalize better and give a higher predictive performance on unseen data. Specifically, by using the MTL approach, the generalization of the model improves if the dataset of each task is expanded by observations from highly related tasks. This is crucial, especially in cases for which the number of training instances per task is limited. The basic idea that underlines the MTL modelling approach is the learning of a separate model for each task and not a unique model trained on a concatenated set of observations of all tasks. Note that in our spatio-temporal datasets, each location can be seen as a different task, and neighbouring (or distant) locations with similar climate-vegetation interactions will tend to have similar (yet not identical) behaviour. In light of this observation, MTL seems to be a quite natural modelling approach to explore the interaction between climate and vegetation in different locations.

The idea of MTL is not new (Baxter, 1997; Caruana, 1997; Baxter, 2000), and it has been applied in many machinelearning applications in medical sciences (Bi et al., 2008; Zhang and Shen, 2012) and computer vision (Zhang et al., 


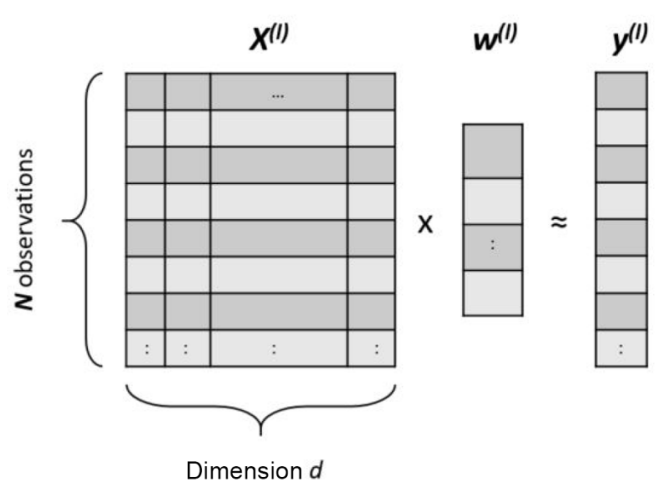

(a)

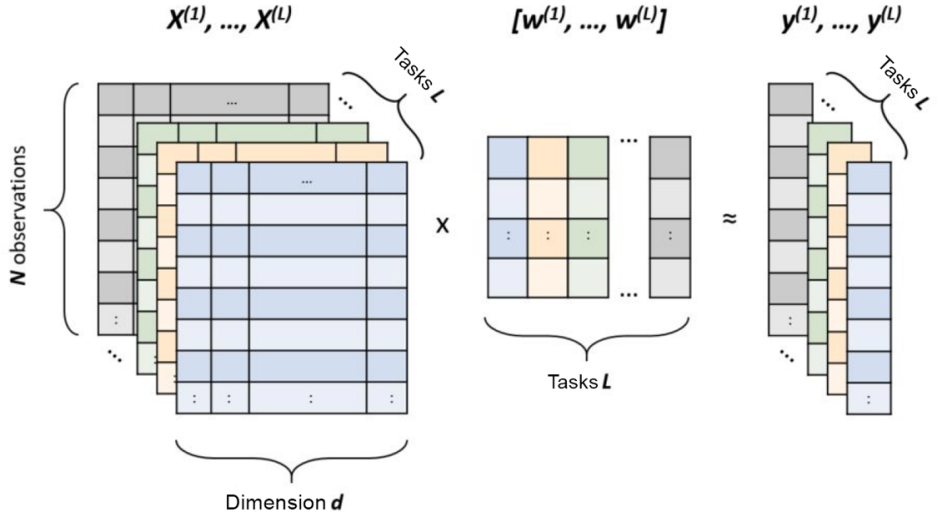

(b)

Figure 1. Graphical representation of two learning approaches. (a) A single-task learning approach in which each pixel is treated separately. For each pixel $l$ there is an input dataset $\mathbf{X}^{(l)} \in \mathbb{R}^{N \times d}$, with $N$ being the number of observations and $d$ being the number of predictors, and a target vector $\boldsymbol{y}^{(l)} \in \mathbb{R}^{N}$. The vector $\boldsymbol{w}^{(l)} \in \mathbb{R}^{d}$ represents the weight vector learned by the model. (b) A multitask learning approach in which the models of $L$ tasks are simultaneously learned. The input of the method is the datasets $\mathbf{X}^{(1)}, \mathbf{X}^{(2)}, \ldots, \mathbf{X}^{(L)}$ of all locations (i.e. all global land pixels). The corresponding target vectors are denoted with $\boldsymbol{y}^{(1)}, \boldsymbol{y}^{(2)}, \ldots, \boldsymbol{y}^{(L)}$. The weight matrix $\left[\boldsymbol{w}^{(1)}, \boldsymbol{w}^{(2)}, \ldots, \boldsymbol{w}^{(L)}\right] \in \mathbb{R}^{d \times L}$ contains the weight vectors for all tasks.

2014). It has also been used in climate science to improve the way multiple Earth system model (ESM) outputs are combined by treating the locations as different tasks (Subbian and Banerjee, 2013; McQuade and Monteleoni, 2013). In these studies, the idea is that in neighbouring locations (pixels which are close to each other), similar ESMs tend to have similar performance. A recent study proposed a hierarchy of tasks, in which at a first level, tasks of each location are trained into an MTL setting, while at a second level, tasks of each variable are sharing information (Gonçalves et al., 2017). In addition, for modelling spatio-temporal data, Xu et al. (2016) introduced an MTL framework in which local models share a common representation based on the spatial autocorrelation. Although this kind of modelling is becoming more common in climate science (e.g. Subbian and Banerjee, 2013; McQuade and Monteleoni, 2013; Gonçalves et al., 2017; Xu et al., 2016), it has not been combined (to the best of our knowledge) with clustering approaches in the context of mapping land cover or climate-vegetation dynamics.

In this work, we focus on MTL methods that can discover the relationship between different tasks (locations) and recover strong predictive structures of the vegetation response to climate. These are then used to conform hydro-climatic biomes, i.e. regions of coherent vegetation behaviour with respect to climate variability (see Sect. 3.3). To this end, we use the same notation as before by denoting $\mathbf{X}^{(l)} \in \mathbb{R}^{N \times d}$ as the input data matrix of the predictor variables, $\boldsymbol{y}^{(l)} \in \mathbb{R}^{N}$ as the target vector for each location $l$, and $\boldsymbol{w}^{(l)} \in \mathbb{R}^{d}$ in which each value corresponds to a weight. We define as $\left[\boldsymbol{w}^{(1)}, \boldsymbol{w}^{(2)}, \ldots, \boldsymbol{w}^{(L)}\right] \in \mathbb{R}^{d \times L}$ as the weight matrix of all locations such that the $\boldsymbol{w}^{(l)}$ vector is the $l$ th column of the $\left[\boldsymbol{w}^{(1)}, \boldsymbol{w}^{(2)}, \ldots, \boldsymbol{w}^{(L)}\right]$ matrix - see a graphical representa- tion of the notation in Fig. 1b. Given a loss function $\mathcal{L}$ (e.g. the squared error loss), the multitask minimization problem is formulated as

$$
\min _{w^{(1)}, \ldots, w^{(L)}} \sum_{l=1}^{L} \sum_{i=1}^{N} \mathcal{L}\left(\boldsymbol{w}^{(l)} \boldsymbol{x}_{i}^{(l)}, y_{i}^{(l)}\right)+\Omega\left(\boldsymbol{w}^{(1)}, \ldots, \boldsymbol{w}^{(L)}\right),
$$

where $\Omega\left(\boldsymbol{w}^{(1)}, \ldots, \boldsymbol{w}^{(L)}\right)$ is a factor which controls the relatedness among the tasks. In our setting, we assume that there is no prior knowledge about the relationship of the tasks (locations) and we aim to apply a method that can discover these relationships.

In the literature, there are many MTL methods that are trying to do two things simultaneously: learn a weight matrix $\left[\boldsymbol{w}^{(1)}, \boldsymbol{w}^{(2)}, \ldots, \boldsymbol{w}^{(L)}\right]$ and another matrix which captures the task relationships simultaneously (Ando and Zhang, 2005; Chen et al., 2009; Zhou et al., 2011). In real applications, there are scenarios in which the tasks of an MTL problem follow a specific structure; i.e. some tasks are more related, whereas some others are unrelated. In order to identify this group structure, researchers have developed various methods which have been referred to as clustered multitask learning (CMTL) methods (Zhou et al., 2011). For instance, Xue et al. (2007) proposed a method which uses a Dirichlet process-based statistical model to identify similarities between related tasks, while Jacob et al. (2009) introduced a framework which identifies groups of tasks and performs the learning at once. In the same direction, Wang et al. (2009) used an inter-task regularization term to take into consideration tasks which have been grouped in the same cluster in a semi-supervised setting. More recently, Barzilai and Crammer (2015) suggested a method which explicitly assigns each 
task to a specific cluster, building a single model for each task by using linear classifiers which are combinations of some basis. An alternative approach has been proposed by Zhou et al. (2011) in which the structure of the task relatedness is unknown and is learned during the training phase. Interestingly, when case-specific conditions are fulfilled, this method is equivalent to the method by Ando and Zhang (2005), known as alternative structure optimization (ASO), which belongs to the category of MTL methods that assume the existence of a shared low-dimensional representation among the tasks. The name of the method indicates that an alternating optimization procedure is involved during the learning process since the weight matrix and the matrix which captures the shared low-dimensional representation are learned simultaneously. Typically, in these procedures, the optimization of each part is separately performed, while the other part remains fixed. In our work, we apply the ASO method due to its simplicity and the fact that it does not need a lot of iterations to capture the information about the task relatedness that is needed. This is crucial for our application, since the large size of the global database we use (Papagiannopoulou et al., 2017a) puts severe limitations on the choice of method. Another aspect is that by learning this low-dimensional representation we can have a visual inspection of the "most predictive common structures" for each region. In the following section we explain in detail the ASO method used in our setting.

\subsection{Learning predictive structures from multiple tasks}

The ASO algorithm proposed by Ando and Zhang (2005) learns common predictive structures from multiple related tasks that are assumed to share a low-dimensional feature space. Specifically, by applying this method, one learns one model function for each individual task and the learned weight vector is decomposed into two parts: (a) a highdimensional space and (b) a shared low-dimensional space based on a feature map learned during the process. This feature map is a matrix which serves as a link between a highdimensional space and a low-dimensional space. In our case, $L$ predictor functions $\left\{f^{(l)}\right\}_{l=1}^{L}$ are simultaneously learned by exploiting the shared feature space that underlines all tasks. This low-dimensional feature space is expressed in the simple linear form of a low-dimensional feature map $\Theta$ across the $L$ tasks. Mathematically, the function $f^{(l)}$ can be written as

$f^{(l)}(\boldsymbol{x})=\boldsymbol{w}^{(l)} \boldsymbol{x}_{i}^{(l)}=\boldsymbol{u}^{(l)} \boldsymbol{x}_{i}^{(l)}+\boldsymbol{v}^{(l)} \boldsymbol{\Theta} \boldsymbol{x}_{i}^{(l)}$,

with $\Theta \in \mathbb{R}^{h \times d}$ being a parameter matrix with orthonormal row vectors; i.e. $\boldsymbol{\Theta \Theta}^{T}=\mathbf{I}$, where $h$ is the dimensionality of the shared feature space, and $\boldsymbol{w}^{(l)}, \boldsymbol{u}^{(l)}$, and $\boldsymbol{v}^{(l)}$ are the weight vectors for the full feature space, the highdimensional one (initial dimension $d$ ), and the shared lowdimensional one (based on the $h$ parameter), respectively. As mentioned before, the ASO method is equivalent to the
CMTL method (Zhou et al., 2011) under a specific condition: that the parameter $k$, which symbolizes the number of clusters in the CMTL approach, is equal to the parameter $h$ of the ASO method. This condition determines the number of clusters that should be used in the clustering phase of our framework because the objective of ASO is optimized based on the value of the parameter $h$. We reconsider this equivalence in Sect. 3.2 where we discuss the number of clusters that should be identified based on our analysis.

Formally, ASO can be formulated as the following optimization problem:

$\min _{\left\{\boldsymbol{w}^{(l)}, \boldsymbol{v}^{(1)}\right\}, \boldsymbol{\Theta} \boldsymbol{\Theta}^{T}=\mathbf{I}} \sum_{l=1}^{L}\left(\sum_{i=1}^{N} \mathcal{L}\left(\boldsymbol{w}^{(l)} \boldsymbol{x}_{i}^{(l)}, y_{i}^{(l)}\right)+\lambda^{(l)}\left\|\boldsymbol{u}^{(l)}\right\|_{2}^{2}\right)$,

with $\left\|\boldsymbol{u}^{(l)}\right\|_{2}^{2}$ being the regularization term $\left(\boldsymbol{u}^{(l)}=\boldsymbol{w}^{(l)}-\mathbf{\Theta}^{T} \boldsymbol{v}^{(l)}\right)$ that controls the task relatedness among $L$ tasks, $\left(\boldsymbol{x}_{i}^{(l)}, y_{i}^{(l)}\right)$ being the input vector and the corresponding target value of the $i$ th observation in a particular location $l$, and $\lambda^{(l)}$ being a predefined parameter - see Fig. 2 for the graphical representation of the notation. During the learning process the weight matrix $\left[\boldsymbol{w}^{(1)}, \boldsymbol{w}^{(2)}, \ldots, \boldsymbol{w}^{(L)}\right]$ and the matrix $\Theta$, which captures the shared low-dimensional representation, are learned simultaneously. The regularization term $\left\|\boldsymbol{u}^{(l)}\right\|_{2}^{2}$, based on the value of the parameter $\lambda$, penalizes the differences between the weights on the initial high-dimensional space and the weights on the low-dimensional space parameterized by $\boldsymbol{\Theta}$.

There are several ways of solving the optimization problem in Eq. (3) (Ando and Zhang, 2005). Our main purpose is to extract the shared feature space $\boldsymbol{\Theta}$ in order to apply a clustering on the low-dimensional feature space. In this feature space, locations with similar predictive structures will be grouped into the same broader region. For this reason, we adopt an ASO algorithm based on singular value decomposition (SVD) as proposed by Ando and Zhang (2005), which achieves good performance even on the first iteration of the method. As mentioned before, this is crucial to our application given the large number of tasks and the highdimensional datasets. The steps of the SVD-based ASO are presented in Algorithm 1.

The SVD-based ASO method can be interpreted as a dimensionality reduction technique applied to the model space (i.e. weights). It should be stressed here that this method must not be confused with PCA, which is usually employed on the data space (input space of predictors) (Metzger et al., 2012; Ivits et al., 2014). The goal of the ASO method is to detect the principal components of the parameter matrix, while PCA identifies the principal components of the input data $\mathbf{X}$. The goal of the ASO method can be achieved by considering the models of multiple tasks as samples of their own distribution. Therefore, these samples can only be formed by using an MTL approach in which there is access to the models from multiple learning tasks. Moreover, in our work, we 


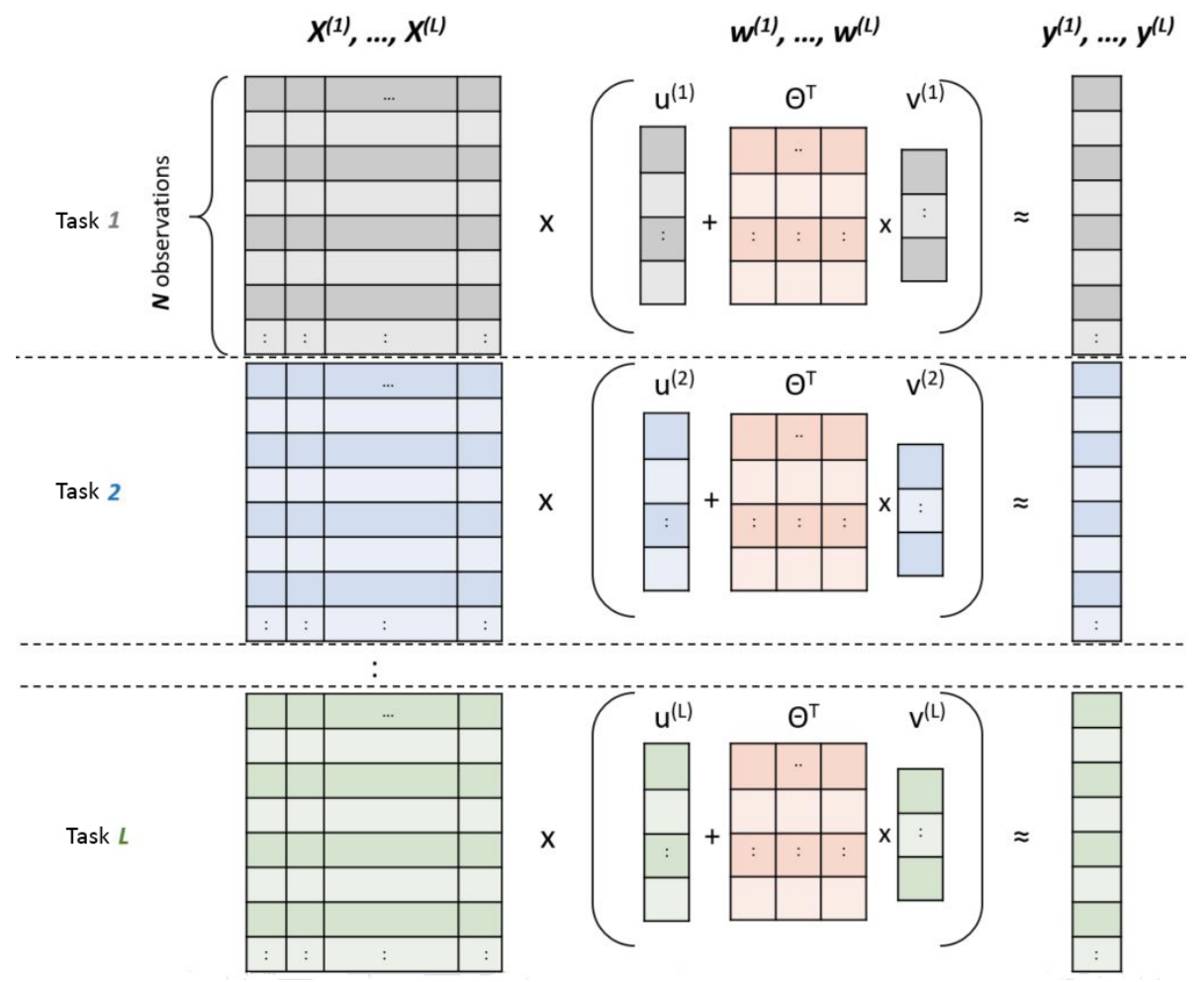

Figure 2. Graphical representation of the ASO method. The input of the method is the datasets $\mathbf{X}^{(1)}, \mathbf{X}^{(2)}, \ldots, \mathbf{X}^{(L)}$ of all locations. The corresponding target vectors are denoted with $y^{(1)}, y^{(2)}, \ldots, y^{(L)}$. The weight vector $\boldsymbol{w}^{(l)} \in \mathbb{R}^{d}$ of the full space is decomposed into two parts: to the weight vector $\boldsymbol{u}^{(l)} \in \mathbb{R}^{d}$ of the high-dimensional space and the weight vector $\boldsymbol{v}^{(l)} \in \mathbb{R}^{h}$ of the low-dimensional one. The lowdimensional feature map $\Theta^{T} \in \mathbb{R}^{d \times h}$ is common for all the tasks.

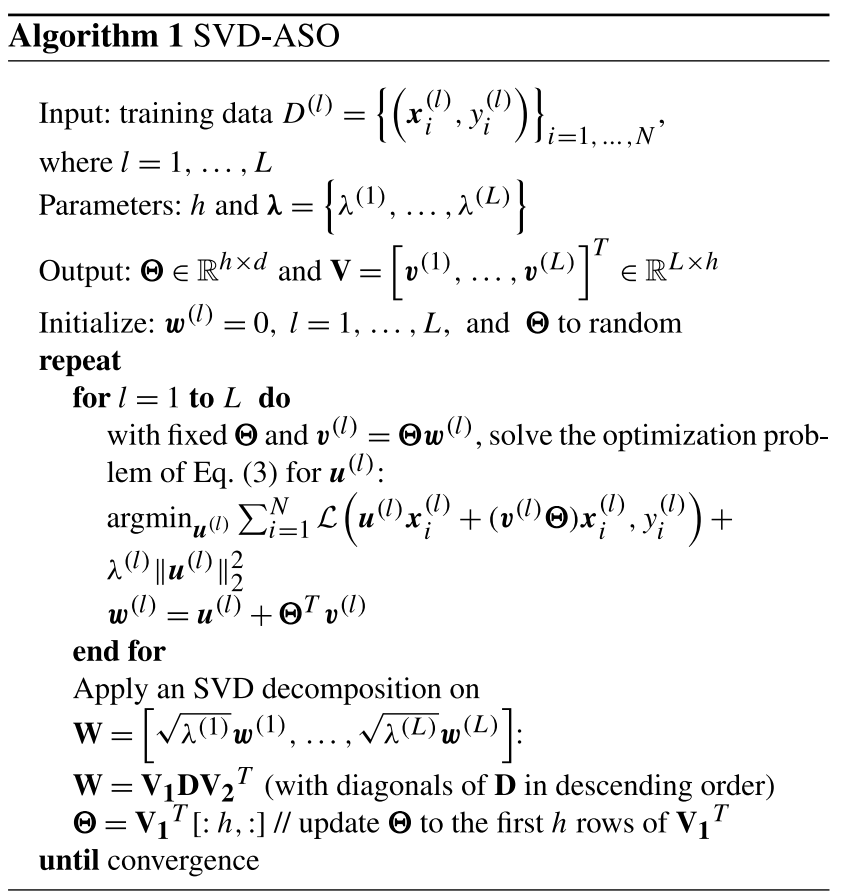

explicitly consider the climatic variables as predictors and the vegetation variable as a target variable, and we learn the relationship between them in a supervised setting. As such, the regions that we define rely on the relationship between climate and vegetation in a prediction setting, and the clustering is calculated based on the similarity of this relationship (i.e. the model coefficients for different locations); see Sect. 2.5 for more details. As such, we learn relationships between climate and vegetation in a supervised setting, whereas PCA-based methods (Metzger et al., 2012; Ivits et al., 2014) are fully unsupervised. In our study the SVD decomposition is used as part of the optimization algorithm and thus in a supervised setting. In this setting, the model weights are optimized based on a given training set. Therefore, the discovered structures are obtained during the training process.

To clarify the notation used in the ASO method, we intuitively explain the symbolization of the method in relation to our specific setting: the problem of detecting locations with similar climate-vegetation dynamics. As mentioned above (Sects. 2.2 and 2.3), the input features that constitute the $\mathbf{X}^{(l)} \in \mathbb{R}^{N \times d}$ matrix consist of the climatic predictor variables, i.e. the extreme indices and lagged variables, calculated based on raw climatic time series of a certain location $l$. The dimensions $N$ and $d$ correspond to the number of ob- 
servations, i.e. the length of the time series and the number of predictor variables, respectively. The target variable for a particular location $l$, which is the NDVI anomalies, is symbolized with $\boldsymbol{y}^{(l)} \in \mathbb{R}^{N}$. As such, an observation of a certain location $l$ at a particular timestamp $i$ is denoted as a pair $\left(\boldsymbol{x}_{i}^{(l)}, y_{i}^{(l)}\right)$. The goal of the ASO method is to learn the weight matrix $\left[\boldsymbol{w}^{(1)}, \boldsymbol{w}^{(2)}, \ldots, \boldsymbol{w}^{(L)}\right]$, i.e. a single weight vector $\boldsymbol{w}^{(l)}$ for each location $l$. This weight vector $\boldsymbol{w}^{(l)}$ is able to capture the relationship between the predictor variables and the target, i.e. the climatic variables and the NDVI anomalies. Therefore, climatic predictors that are more important for vegetation anomalies correspond to higher absolute values in the weight vector $\boldsymbol{w}^{(l)}$. As a result, locations with similar weights are considered as regions where vegetation responds to climate in a similar way. As described in a previous paragraph of this section, the ASO method assumes that the weight vectors $\boldsymbol{w}^{(l)}$ consist of two parts: the $\boldsymbol{u}^{(l)}$ and the $\boldsymbol{v}^{(l)} \boldsymbol{\Theta}$. These two parts are learned simultaneously in Algorithm 1 in an alternating fashion. The first part, i.e. the $\boldsymbol{u}^{(l)} \in \mathbb{R}^{d}$, belongs to the high-dimensional space, the initial one, which is equal to $d$. This part expresses the locationspecific part of the weight vector, i.e. the deviation of each location's weight vector from the weights learned in a lower dimensional space. The second part consists of the matrix $\Theta \in \mathbb{R}^{h \times d}$ that represents the map from the initial dimension $d$ to the lower dimension $h$ and the weight vector $\boldsymbol{v}^{(l)} \in \mathbb{R}^{h}$. The map matrix $\Theta$ is common for all the locations (tasks) and can be learned across them due to the MTL approach. The weight vector $\boldsymbol{v}^{(l)}$ represents the projection of the initial weights to a low-dimensional space $h$. Intuitively, this second part of the weight decomposition expresses the coarsest and most important part of the weights, since it detects the most important structures through the map matrix $\boldsymbol{\Theta}$. The matrix $\mathbf{V}=\left[\boldsymbol{v}^{(1)}, \ldots, \boldsymbol{v}^{(L)}\right]^{T} \in \mathbb{R}^{L \times h}$ denotes the representation of the models in the low-dimensional space $h$ for the $L$ locations.

\subsection{Land classification: clustering highly predictive structures}

Clustering in machine learning is the task of grouping a set of samples in such a way that those samples that belong to the same group (cluster) are more similar with respect to a specific criterion than to samples that belong to other groups. Clustering techniques are usually based on a distance (or similarity) measure, which is calculated among the samples and/or group of samples. There are several clustering approaches and an in-depth review can be found in $\mathrm{Xu}$ and Tian (2015).

It is known that in high-dimensional spaces, distance measures are not able to capture the differences between pairs of samples well, and thus clustering algorithms tend to perform better in lower dimensional spaces. In our setting, we learn the common feature map $\boldsymbol{\Theta} \in \mathbb{R}^{h \times d}$ and the
$\mathbf{V}=\left[\boldsymbol{v}^{(1)}, \ldots, \boldsymbol{v}^{(L)}\right]^{T} \in \mathbb{R}^{L \times h}$ matrix, which is the representation of the models in this low-dimensional space, using the SVD-ASO method - see Sect. 2.4. The $\mathbf{V}$ matrix captures the information of the similar predictive structures among all the tasks, so similar tasks are closer in this low-dimensional space and as a consequence they have a similar representation (i.e. weights) in this matrix. That way, the clustering techniques based on distance calculations are applied on the more expressive low-dimensional space, resulting in a better performance. As has been discussed in our previous work (Papagiannopoulou et al., 2017a), global climate-vegetation relationships are complex and non-linear. Here, if the $\mathbf{V}$ representation is expressive enough, the clustering method can group together locations with similar models, i.e. locations in which vegetation responds to climate in a similar non-linear way. Thus, it is first necessary to evaluate the quality of the learned matrix $\mathbf{V}$. The most straightforward way to do so is by measuring the predictive performance of the MTL model in terms of e.g. $R^{2}$. If the predictive power of the model is strong, we can conclude that the $\mathbf{V}$ matrix is able to capture the relationships of each task well with the highly predictive structures. So, given that the $\mathbf{V}$ representation is sufficiently learned from the data, we can apply any kind of clustering algorithm on the low-dimensional representation of matrix V. This approach is also known as spectral clustering due to the fact that the clustering algorithm is applied on a reduced feature space, making the clustering results more robust.

In our application, we use a hierarchical agglomerative clustering approach (Ward, 1963) in which the number of clusters is not predefined. In the hierarchical clustering approach, the result is usually depicted as a dendrogram in which the leaves represent the observations and the inner nodes correspond to the data clusters. The dendrogram branches are proportionally long to the value of the intergroup dissimilarity. By defining this hierarchical form of the clustering result, one can define the number of clusters by cutting down vertically (or horizontally, depending on the view) the dendrogram in a point at which the dissimilarity between the clusters is high and therefore the branches are longer - see Sect. 3.2 for the choice of the optimum number of clusters in our analysis.

\subsection{Experimental set-up}

In all the experiments, we use as predictors all the climatic datasets and the features that we have constructed from them as well as the 12 lagged values of the target variable. A resulting number of 3209 predictor (climate) variables is used; i.e. $d=3209$ in our setting. These variables constitute the input to our framework, i.e. the $\mathbf{X}^{(l)}, l=1, \ldots, L$ datasets. As the target variable, we use the NDVI seasonal anomalies calculated as in Papagiannopoulou et al. (2017a) and denoted as $\boldsymbol{y}^{(l)}, l=1, \ldots, L$ for each location. For more details about the datasets in our setting see Sect. 2.1. We examine 13072 land pixels with each pixel constituting a single task in our 
MTL setting; i.e. $L=13072$. The dataset of each single task consists of 360 monthly observations given our 30 -year study period; i.e. $N=360$.

For the STL modelling, evaluated for comparison, we use the ridge regression for each location independently. Ridge regression is a linear model which uses an $\ell_{2}$ norm regularization term in order to shrink the weight coefficients towards zero and avoid over-fitting. In ridge regression the weight coefficients are fitted by solving the following optimization problem:

$\min _{\boldsymbol{w}^{(l)}} \sum_{i=1}^{N} \mathcal{L}\left(\boldsymbol{w}^{(l)} \boldsymbol{x}_{i}^{(l)}, y_{i}^{(l)}\right)+\lambda\left\|\boldsymbol{w}^{(l)}\right\|^{2}$,

with $\lambda$ being a regularization parameter tuned using a separate validation set and $\left\|\boldsymbol{w}^{(l)}\right\|^{2}$ being a penalty term, i.e. the squared $\ell_{2}$ norm of the weight vector. Note that by splitting the original dataset into three parts - (1) training set, (2) validation set, and (3) test set - we tune the parameters in a set of observations (validation set) that are not included in the final test set and achieve a fair evaluation of the model performance. The optimization problems of the SVD-ASO algorithm are solved by using the limitedmemory Broyden-Fletcher-Goldfarb-Shanno (L-BFGS) optimization algorithm.

\section{Results and discussion}

\subsection{Single-task versus multitask learning model}

In a first experiment, we compare the predictive performance of the STL model versus the MTL model. For the STL modelling, ridge regression is used. For the MTL modelling, we apply the ASO-MTL model (Ando and Zhang, 2005) described in Sect. 2. We use a separate validation set to tune the regularization parameter $\lambda$ for both approaches. For the STL approach, we tune the $\lambda$ parameter for each location (task) separately, while for the MTL approach we use the same $\lambda$ value for all the tasks, taking into account the average performance across these tasks. For the ASO-MTL method, we have also experimented with the value of the $h$ parameter, which is the dimensionality of the shared feature space - see Sect. 3.2 for more details about the influence of this parameter on the clustering results. Finally, we evaluate the performance of both approaches in terms of $R^{2}$, as in Papagiannopoulou et al. (2017a). Figure 3 depicts the result of our comparison. Figure 3a shows the $R^{2}$ of the ASO-MTL model, while Fig. $3 \mathrm{~b}$ highlights the difference in predictive performance of the MTL model in comparison with the STL model. As shown in Fig. 3b, in almost all regions of the world, the predictive performance increases substantially compared to the STL approach. In fact, over extensive regions ( $40 \%$ of the study area), more than $5 \%$ of the variability in NDVI is explained by the spatial structure of the data. In statistical terms, this implies the existence of a hidden structure between the different locations (tasks), which is informative with respect to our target variable. The dotted regions in Fig. $3 \mathrm{~b}$ correspond to areas where the MTL model significantly outperforms the STL models based on the Diebold-Mariano statistical test, which compares model predictions (Diebold, 2015). For the statistical test, we use the false discovery rate (Benjamini and Hochberg, 1995) method to correct the $p$ values at level 0.05 due to the multiple-hypothesis testing setting.

Additionally, Fig. 3a shows that more than $40 \%$ of the mean monthly vegetation dynamics can be explained by climate variability in some regions. In particular, in regions such as Australia, Africa, and Central and North America, the predictive power of the model is stronger in terms of $R^{2}$, following the same pattern and scoring similar $R^{2}$ values as the random forest approach by Papagiannopoulou et al. (2017a). To elaborate on the performance difference between the two approaches, the $R^{2}$ scores are presented as two different distributions in Fig. 3c. The blue histogram corresponds to the distribution of the $R^{2}$ scores of the STL approach, while the orange one corresponds to the distribution of the $R^{2}$ scores of the MTL approach. As can be observed, the distribution of the $R^{2}$ scores is shifted to the right for the MTL, meaning that values are typically greater than those derived from the STL approach. Moreover, the skew towards the left in the blue histogram, with values close to zero, is an indication of the near-zero performance of the STL models in many locations. The Wilcoxon paired statistical test (Demšar, 2006) confirms that the results of the two approaches are overall statistically different ( $p$ value $<10^{-9}$ ).

Since we are ultimately interested in investigating regions of coherent impact of climate variability on vegetation dynamics, we also evaluate the ability of the MTL model to detect Granger causal effects of climate on vegetation. For a detailed description of the Granger causality modelling framework we direct the reader to Papagiannopoulou et al. (2017a). This point is crucial to understand the extent to which the climatic predictors carry additional information about the dynamics in vegetation that are not contained in the past vegetation signal itself. The results of applying the Granger causality analysis using MTL modelling are shown in Fig. 3d, which illustrates the results of the full MTL model compared to the baseline MTL model. This baseline model only uses previous values of NDVI to predict monthly NDVI anomalies (Papagiannopoulou et al., 2017a). In this figure it becomes clear that climate dynamics "Granger-cause" monthly vegetation anomalies in most regions of the world, and the ability of the MTL model to detect deterministic relationships is evidenced. This is also confirmed by the Wilcoxon paired statistical test ( $p$ value $<10^{-9}$ ). On the other hand, the ability of the STL model to detect Granger causal relationships is rather limited compared to that of the MTL model. Figure 3e depicts the result of the comparison; in almost all regions the quantification of the Granger causality of the MTL approach increases substantially compared to the one of the STL ap- 

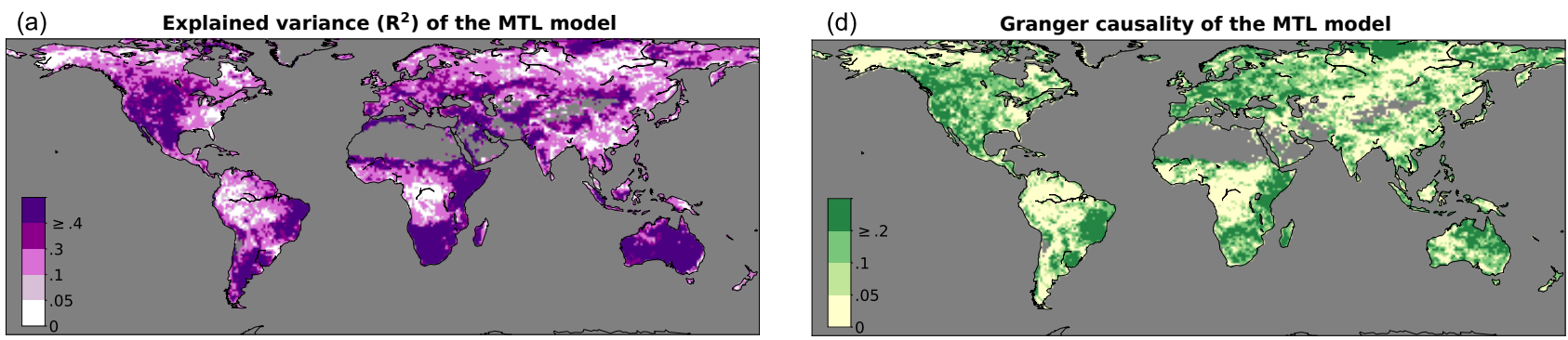

(b) Difference $\left(R^{2}\right)$ between MTL and STL models
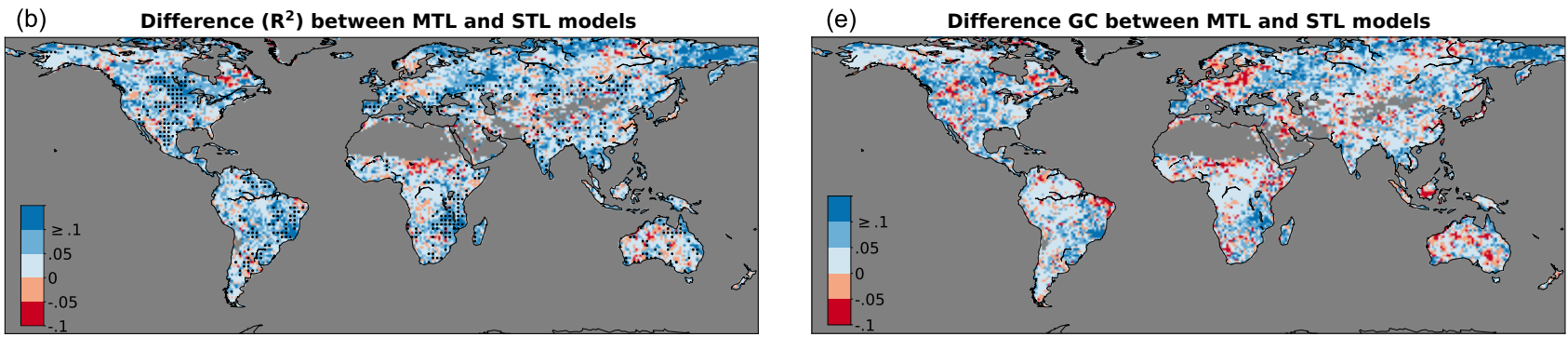

(c)

(f)
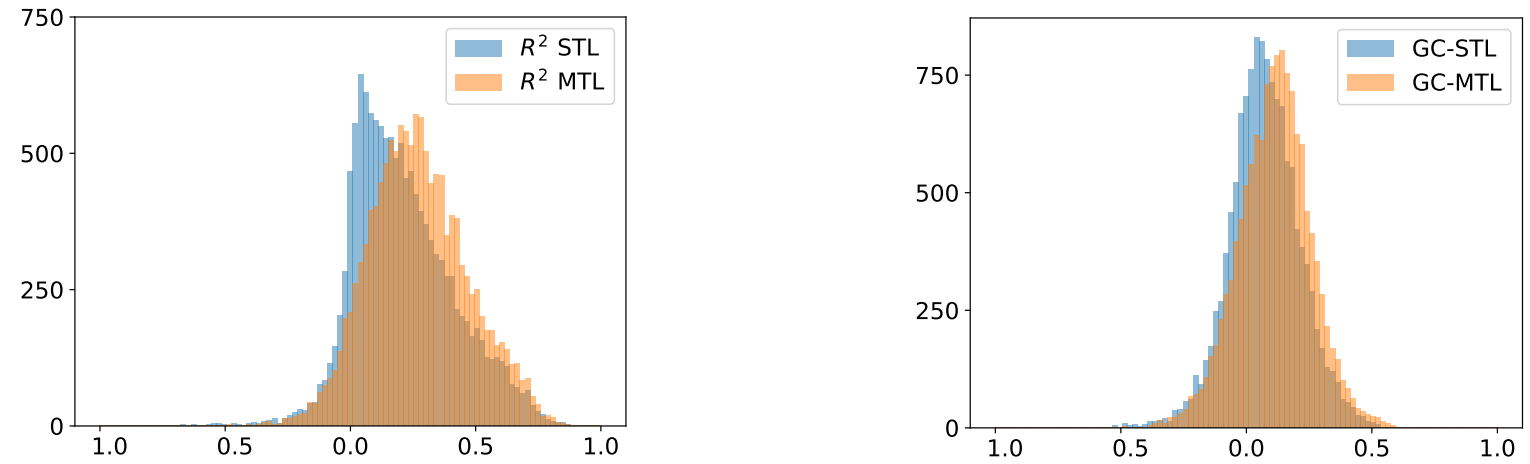

Figure 3. Comparison of the predictive performance between the STL and the MTL approaches. (a) Explained variance $\left(R^{2}\right)$ of the NDVI monthly anomalies based on the MTL approach. (b) Difference in terms of $R^{2}$ between the MTL and the STL approaches; blue regions indicate a higher performance by the MTL. The dotted regions correspond to areas where the MTL model significantly outperforms the STL models based on the Diebold-Mariano statistical test (Diebold, 2015). (c) Comparison of the distributions of the $R^{2}$ scores in the STL and in the MTL setting; the blue histogram corresponds to the STL, and the orange one to the MTL approach. (d) Quantification of Granger causality for the MTL approach, i.e. improvement in terms of $R^{2}$ by the full MTL model with respect to the $R^{2}$ of the baseline MTL model that uses only past values of NDVI anomalies as predictors; positive values indicate Granger causality (Papagiannopoulou et al., 2017a). (e) Difference in terms of Granger causality between the MTL and the STL approaches; blue regions indicate a higher performance by the MTL. (f) Comparison of the distributions of Granger causality in the STL and in the MTL setting; the blue histogram corresponds to the STL, and the orange one to the MTL approach.

proach. Analogous to Fig. 3c, Fig. $3 f$ compares the distributions of Granger causality (i.e. the difference in predictive performance in terms of $R^{2}$ between the full and the baseline model) between the STL and MTL approach. Once again, the blue histogram corresponds to the distribution of Granger causality retrieved using the STL approach, while the orange corresponds to the results of the MTL approach. The shift to the right of the orange histogram shows the larger ability of the MTL model to reveal Granger causality between climate and vegetation. Similar to the previous comparison, the Wilcoxon paired statistical test (Demšar, 2006) confirms that the results of the two approaches are overall statistically different ( $p$ value $<10^{-9}$ ). In summary, these findings highlight the potential of using the low-dimensional feature representation learned from the data to fulfill our final objective, which is the detection of vegetated areas holding a similar response to climate via a clustering approach.

\subsection{Appropriate number of hydro-climatic biomes}

As described in Sect. 2.5, there are multiple approaches that can be used to define the number of classes in a clustering 
problem. In our framework, we define the number of clusters by using a data-driven approach. In our analysis, we choose not to use information from any predefined number of vegetation and/or climate classes existing in the literature, since the ultimate goal is to identify land classes fully independently and only based on the observed relationship between vegetation and climate. To this end, we rely on the definition of the number of clusters on the predictive performance of the MTL model. In Sect. 2.3, it is stated that the ASO-MTL approach shares the objective function of the CMTL method. This only holds if the number of clusters (which is a predefined parameter in the CMTL method) is equal to the value of the parameter $h$ in the ASO-MTL method, which is the dimensionality of the common feature space. In light of this equivalence relation, we experimented with a wide range of values for $h$ in a validation set, aiming to select the value of $h$ that maximizes the model performance in terms of $R^{2}$. As such, we conclude that the best predictive performance occurs at $h=11$ and that the appropriate number of biomes in the clustering phase equals 11 - see Sect. S2 for more details.

The results of this hierarchical clustering (with Euclidean distance) can be visualized in a dendrogram representation, which provides an indication about the optimal number of clusters that emerge from the dataset. Figure $4 \mathrm{~b}$ depicts the dendrogram formed by our framework, with the vertical cutting line separating the data into 11 clusters. This representation allows for a visual inspection of whether the choice of 11 clusters is in line with the dissimilarities in the observations. As one can observe, our choice is reasonable, since the clusters at this point are quite dissimilar, based on the Euclidean distance metric, compared to hypothesized cutting lines either before or after this point. In other words, the branches of the dendrogram are already quite long at 11 clusters, indicating high dissimilarities between the resulting classes.

\subsection{Hydro-climatic biomes}

The final objective of this study is to uncover the regions in which vegetation responds in a analogous way to climate anomalies, here referred to as "hydro-climatic biomes". In the previous section, we investigated the appropriate number of such regions based on the information contained in our database. Figure 4a illustrates the spatial distribution of the emerging global hydro-climatic biomes. The colours depicted correspond to those of the clusters in the dendrogram of Fig. 4b. Further analysis of this dendrogram, in combination with the spatial distribution of the clusters in Fig. 4a, shows that our framework can clearly differentiate the bioclimatic behaviour of northern latitude ecosystems from those in middle and southern latitudes. The behaviour of tropical ecoregions is unsurprisingly closer to the behaviour of subtropical ones, while boreal regions sharing exposure to low-temperature anomalies have a more coherent response to one another, forming the second main branch of the dendrogram. Bearing in mind the results of the Granger causality approach by Papagiannopoulou et al. (2017b), as well as the prior knowledge on climate and land use classification, we define the hydro-climatic biomes as follows: (1) tropical, (2) transitional water-driven, (3) transitional energy-driven, (4) subtropical energy-driven, (5) subtropical water-driven, (6) mid-latitude water-driven, (7) mid-latitude temperature-driven, (8) boreal temperature-driven, (9) boreal water-driven, (10) boreal water-temperature-driven, and (11) boreal energy-driven. This nomenclature is broadly based on latitude and main climatic drivers.

Figure $4 \mathrm{c}$ shows the 10 main climate regions of the Köppen-Geiger climate classification, which is based on precipitation and temperature and their seasonality. On the other hand, the International Geosphere-Biosphere Program (IGBP) (Loveland and Belward, 1997) land cover classification, depicted in Fig. 4d, is mostly based on plant functional types. Without the need to prescribe any land cover or climate classification and only relying on the spatial coherence in the vegetation response to climate anomalies, our hydro-climatic biomes in Fig. 4a clearly depict some of the main characteristic patterns from these traditional classification schemes. For instance, the region of North Asia is quite coherent in terms of climate based on the 10 climate classes shown here (Fig. 4c), but quite diverse in terms of vegetation type (Fig. 4d); the hydro-climatic biomes show a clear distinction in the transition from shrublands (energydriven) to coniferous forests (energy- and water-driven). In North America, the more energy-limited ecosystems along the coasts emerge from the water-driven regions inland, and a latitudinal behaviour is also depicted, partly reflecting the transition from croplands and grasslands into temperate and boreal forests. Patterns in the tropics clearly differentiate between rainforest and transitional savannas, and in South America the different drivers of vegetation dynamics in the arc of deforestation lead to a class change that is depicted by neither the Köppen-Geiger climate classification nor the IGBP land cover classes. Finally the patterns found for arid and warm semi-arid regions (here referred to as "subtropical water-driven") and their transition towards wetter and more vegetated ecosystems agree with the expectations based on vegetation (Fig. 4d) and climate (Fig. 4c).

The comparison to the Köppen-Geiger and IGBP maps serves only as a general evaluation or proof of concept for our hydro-climatic biomes map, since in the end such maps are based on a different rationale and thus there is no intent to "outperform" these classification schemes. However, it can be observed in this comparison that the hydro-climatic biomes map in Fig. 4a combines information on climate and vegetation zones by illustrating regions where vegetation similarly interacts with the multi-month dynamics in climatic and environmental conditions. This conclusion is confirmed by the scatter plots in Fig. 4e-g. Figure 4e depicts our hydroclimatic biomes of Fig. 4a in a climate space of mean annual temperature against precipitation, while Fig. $4 \mathrm{f}$ shows the same but for the Köppen-Geiger climate classes of Fig. 4c. In 
(a)

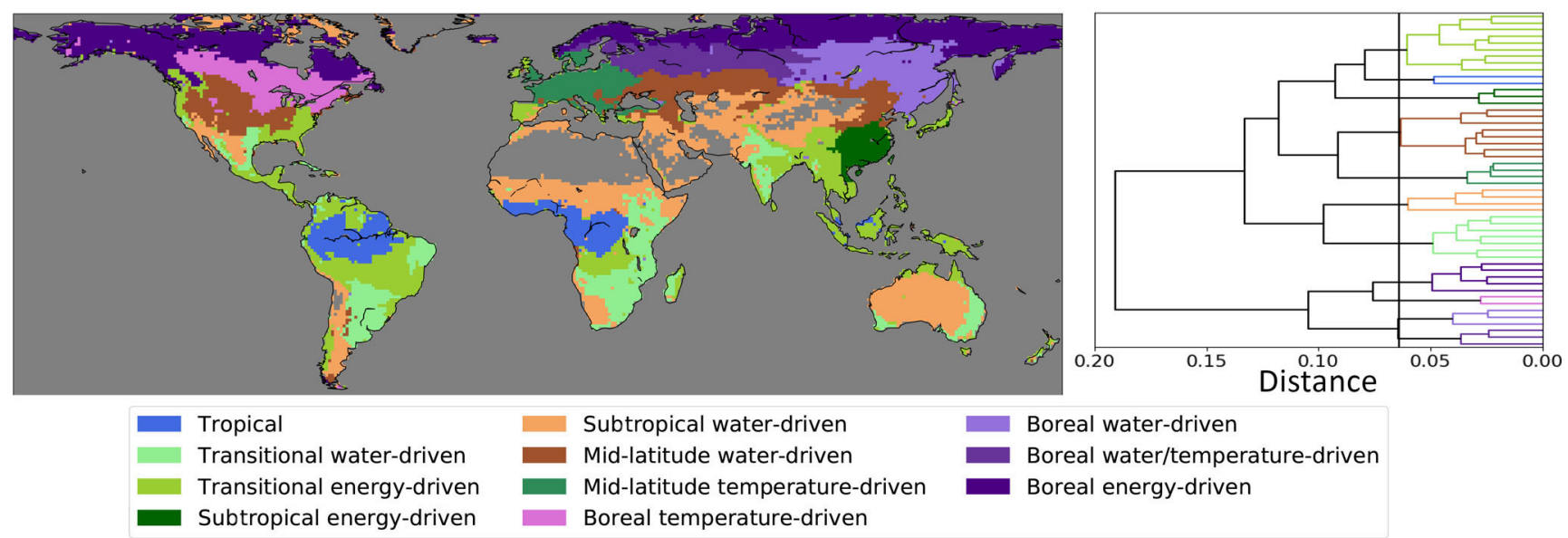

(c)

(d)
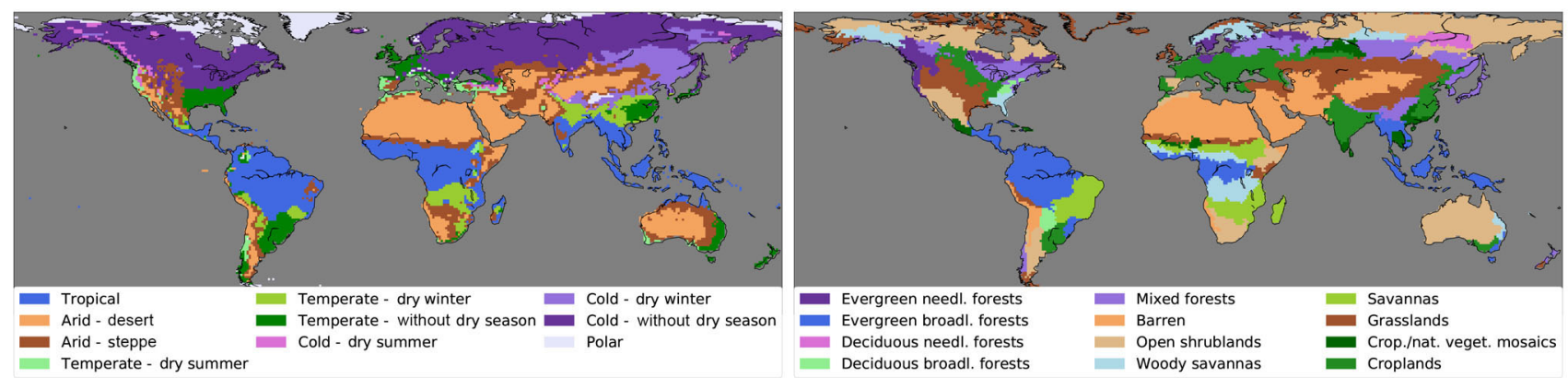

(e)

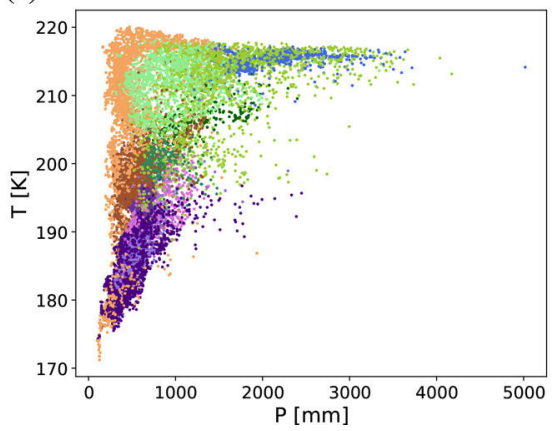

(f)

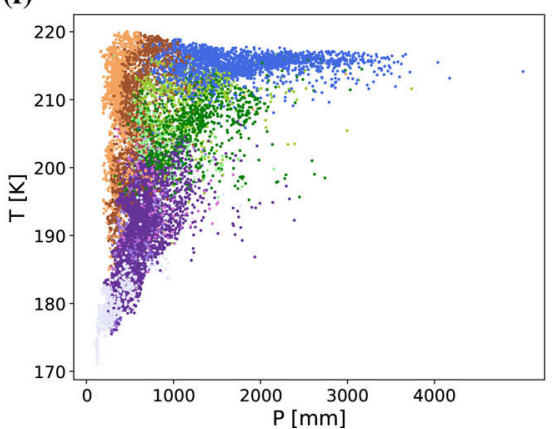

(g)

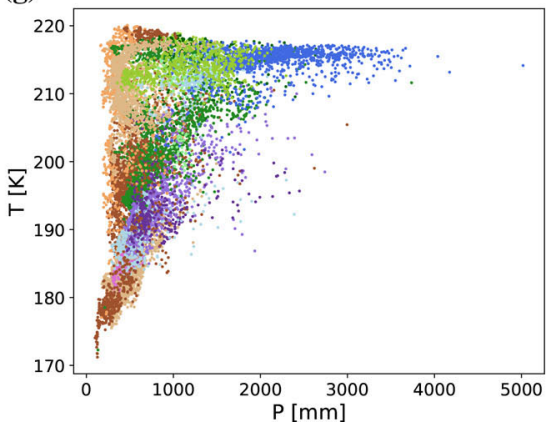

Figure 4. Comparison of the different land surface classification schemes. (a) Hydro-climatic biomes derived from the proposed framework. The region colours correspond to the colours of the clusters that are depicted in the dendrogram. (b) Dendrogram scheme of the clustering derived from the hierarchical agglomerative clustering on the low-dimensional representation of our model observations. The length of the dendrogram branches is a function of the inter-cluster dissimilarities. The vertical cutting line marks the data split into 11 clusters. The denomination of the different classes is supported by the results from Papagiannopoulou et al. (2017b). (c) Simplified Köppen-Geiger climate classification scheme. (d) IGBP land use classification scheme. (e) Climate space (i.e. mean annual temperature versus precipitation) for our hydro-climatic biomes in Fig. 4a. (f) Same as (e) but for the Köppen-Geiger climate classes in Fig. 4c. (g) Same as (e) but for IGBP in Fig. 4d.

Fig. 4f, the five climate classes are well separated, since their definition is based on these two climatic variables. On the other hand, Fig. $4 \mathrm{~g}$ depicts the same information but for the IGBP map of Fig. 4d. In this figure, savannas, tropics, and shrublands appear again well clustered. It can be observed that the scatter plot of Fig. 4e clearly lies between the two previous classifications in terms of clustering. Boreal biomes correspond to cold climate classes, and the subtropical and mid-latitude water-driven biomes correspond to arid regions, while the transitional biomes correspond to the savannas and 
croplands. The clustering of biomes is also consistent with the global distribution of key climatic drivers reported by $\mathrm{Pa}$ pagiannopoulou et al. (2017b) based on random forests and a Granger causality framework, since these biomes are ultimately defined based on the response of vegetation to climatic and environmental conditions. These common dynamics are identified by latent structures in our MTL approach; a discussion on these latent structures is included in Sect. S3. Moreover, we should note that the approach of spectral clustering applied here allows for a robust result, as small perturbations in the datasets do not affect the overall clustering result. This conclusion is confirmed by the fact that even in tropical regions, where the uncertainty in the observations is typically larger and the skill of the predictions is lower (see Fig. 3), the different clusters are separated in a clear manner. A discussion about the comparison of the three land surface classification schemes (the hydro-climatic biomes, the Köppen-Geiger climate classification, and the IGBP land use classification) is presented in Sect. S4. Results for microwave vegetation optical depth (VOD) (Liu et al., 2011) anomalies as an alternative to NDVI anomalies are consistent as shown in Fig. S7 in the Supplement.

\section{Conclusion}

In this paper we introduced a novel framework for identifying regions with similar biosphere-climate interplay dynamics. Our framework combines a multitask learning (MTL) modelling approach and a spectral clustering technique, and it is applied to a global database of global observational climate records compiled by Papagiannopoulou et al. (2017a). Comparisons to a typical single-task learning approach, in which each task (in each location) is analysed separately, indicate that learning about climate-vegetation relationships in neighbouring or even remote locations can help predict local vegetation dynamics based on climate variability. Moreover, our approach is able to detect shared hidden predictive structures among the tasks that enhance the performance of the models. These predictive structures form the basis to which the clustering algorithm is applied to detect regions where vegetation responds to climate in a similar way. We demonstrate that, without the need to prescribe any land cover information, our method is able to identify coherent climate-vegetation interaction zones that emerge directly from the spatio-temporal variability in the data. These zones agree with traditional global classification maps, such as the Köppen-Geiger climate classification or the IGBP land cover classification. We refer to these regions as "hydro-climatic biomes". These wide regions can be used for various applications in geosciences, such as unravelling anomalous relationships between climate and vegetation dynamics at local scales, defining extreme values of vegetation response to climate, exploring tipping points and turning points (Horion et al., 2016) of ecosystem resilience, and benchmarking the dynamic response of vegetation in Earth system models.

Code and data availability. We use the implementation of Python for the L-BFGS optimizer, the singular value decomposition method, and hierarchical clustering (Scikit-learn Python library; Pedregosa et al., 2011). The code for the ASOMTL method (https://doi.org/10.5281/zenodo.1241047) has been uploaded to our GitHub repository (https://github.com/lhwm/ hydro-climatic-biomes, last access: 20 September 2018). Data used in this paper can be accessed using http://www.SAT-EX.ugent.be (last access: 20 September 2018) as a gateway.

\section{The Supplement related to this article is available online at: https://doi.org/10.5194/gmd-11-4139-2018- supplement}

Author contributions. CP, WW, and DGM conceived the study. CP conducted the analysis. CP, DGM, and MD led the writing. All coauthors contributed to the design of the experiments, discussion and interpretation of the results, and editing of the paper.

Acknowledgements. This work is funded by the Belgian Science Policy Office (BELSPO) in the framework of the STEREO III programme, project SAT-EX (SR/00/306). Diego G. Miralles acknowledges support from the European Research Council (ERC) under grant agreement no. 715254 (DRY-2-DRY). The authors thank Stijn Decubber for fruitful discussions. The authors also sincerely thank the individual developers of the wide range of global datasets used in this study. Finally, the authors thank the reviewers for their constructive feedback.

Edited by: David Topping

Reviewed by: Stephanie Horion and one anonymous referee

\section{References}

Ando, R. K. and Zhang, T.: A framework for learning predictive structures from multiple tasks and unlabeled data, J. Mach. Learn. Res., 6, 1817-1853, 2005.

Baker, B., Diaz, H., Hargrove, W., and Hoffman, F.: Use of the Köppen-Trewartha climate classification to evaluate climatic refugia in statistically derived ecoregions for the People's Republic of China, Climatic Change, 98, 113, https://doi.org/10.1007/s10584-009-9622-2, 2009.

Bartholomé, E. and Belward, A. S.: GLC2000: a new approach to global land cover mapping from Earth observation data, Int. J. Remote Sens., 26, 1959-1977, 2005.

Barzilai, A. and Crammer, K.: Convex multi-task learning by clustering, in: Artificial Intelligence and Statistics, San Diego, California, USA, 9-12 May 2015, 65-73, 2015.

Baxter, J.: A Bayesian/information theoretic model of learning to learn via multiple task sampling, Mach. Learn., 28, 7-39, 1997. 
Baxter, J.: A model of inductive bias learning, J. Artif. Intell. Res., 12, 149-198, https://doi.org/10.1613/jair.731, 2000.

Benjamini, Y. and Hochberg, Y.: Controlling the False Discovery Rate: A Practical and Powerful Approach to Multiple Testing, J. Roy. Stat. Soc. B Met., 57, 289-300, 1995.

Bi, J., Xiong, T., Yu, S., Dundar, M., and Rao, R. B.: An improved multi-task learning approach with applications in medical diagnosis, in: Joint European Conference on Machine Learning and Knowledge Discovery in Databases, Springer, 117-132, 2008.

Brugger, K. and Rubel, F.: Characterizing the species composition of European Culicoides vectors by means of the Köppen-Geiger climate classification, Parasite. Vector., 6, 333, https://doi.org/10.1186/1756-3305-6-333, 2013.

Brunsdon, C., Fotheringham, A. S., and Charlton, M. E.: Geographically Weighted Regression: A Method for Exploring Spatial Nonstationarity, Geogr. Anal., 28, 281-298, https://doi.org/10.1111/j.1538-4632.1996.tb00936.x, 1996.

Caruana, R.: Multitask Learning, Mach. Learn., 28, 41-75, https://doi.org/10.1023/A:1007379606734, 1997.

Chan, D. and $\mathrm{Wu}, \mathrm{Q} .:$ Significant anthropogenic-induced changes of climate classes since 1950, Sci. Rep., 5, 13487, https://doi.org/10.1038/srep13487, 2015.

Chen, D. and Chen, H. W.: Using the Köppen classification to quantify climate variation and change: an example for 1901-2010, Environmental Development, 6, 69-79, 2013.

Chen, J., Tang, L., Liu, J., and Ye, J.: A convex formulation for learning shared structures from multiple tasks, in: 26th Annual International Conference on Machine Learning, Montreal, Quebec, Canada, 14-18 June 2009 ACM, 137-144, 2009.

Chen, S. and Tian, Y.: Pyramid of spatial relatons for scene-level land use classification, IEEE T. Geosci. Remote, 53, 1947-1957, 2015.

Congalton, R. G., Gu, J., Yadav, K., Thenkabail, P., and Ozdogan, M.: Global land cover mapping: A review and uncertainty analysis, Remote Sens., 6, 12070-12093, 2014.

De Keersmaecker, W., Lhermitte, S., Tits, L., Honnay, O., Somers, B., and Coppin, P.: A model quantifying global vegetation resistance and resilience to short-term climate anomalies and their relationship with vegetation cover, Global Ecol. Biogeogr., 24, 539-548, https://doi.org/10.1111/geb.12279, 2015.

Demšar, J.: Statistical comparisons of classifiers over multiple data sets, J. Mach. Learn. Res., 7, 1-30, 2006.

Diaz, H. F. and Eischeid, J. K.: Disappearing "alpine tundra” Köppen climatic type in the western United States, Geophys. Res. Lett., 34, L18707, https://doi.org/10.1029/2007GL031253, 2007.

Diebold, F. X.: Comparing Predictive Accuracy, Twenty Years Later: A Personal Perspective on the Use and Abuse of Diebold-Mariano Tests, J. Bus. Econ. Stat., 33, 1-1, https://doi.org/10.1080/07350015.2014.983236, 2015.

Faghmous, J. H. and Kumar, V.: A Big Data Guide to Understanding Climate Change: The Case for Theory-Guided Data Science, Big Data, 2, 155-163, https://doi.org/10.1089/big.2014.0026, 2014.

Feddema, J. J.: A Revised Thornthwaite-Type Global Climate Classification, Phys. Geogr., 26, 442-466, https://doi.org/10.2747/0272-3646.26.6.442, 2005.

Feddema, J. J., Oleson, K. W., Bonan, G. B., Mearns, L. O., Buja, L. E., Meehl, G. A., and Washington, W. M.: Atmospheric science: The importance of land-cover change in simulating future climates, Science, 310, 1674-1678, https://doi.org/10.1126/science.1118160, 2005.

Gallardo, C., Gil, V., Hagel, E., Tejeda, C., and de Castro, M.: Assessment of climate change in Europe from an ensemble of regional climate models by the use of Köppen-Trewartha classification, Int. J. Climatol., 33, 2157-2166, 2013.

Garcia, R. A., Cabeza, M., Rahbek, C., and Araújo, M. B.: Multiple dimensions of climate change and their implications for biodiversity, Science, 344, 1247579, https://doi.org/10.1126/science.1247579, 2014.

Georganos, S., Abdi, A. M., Tenenbaum, D. E., and Kalogirou, S.: Examining the NDVI-rainfall relationship in the semi-arid Sahel using geographically weighted regression, J. Arid Environ., 146, 64-74, https://doi.org/https://doi.org/10.1016/j.jaridenv.2017.06.004, 2017.

Gonçalves, A. R., Banerjee, A., and Von Zuben, F. J.: Spatial Projection of Multiple Climate Variables Using Hierarchical Multitask Learning, in: AAAI Conference on Artificial Intelligence, 4-9 February 2017, San Francisco, California, USA, AAAI, 45094515, 2017.

Hanf, F., Körper, J., Spangehl, T., and Cubasch, U.: Shifts of climate zones in multi-model climate change experiments using the Köppen climate classification, Meteorol. Z., 21, 111-123, 2012.

Herrando-Pérez, S., Delean, S., Brook, B. W., Cassey, P., and Bradshaw, C. J.: Spatial climate patterns explain negligible variation in strength of compensatory density feedbacks in birds and mammals, PLoS One, 9, e91536, https://doi.org/10.1371/journal.pone.0091536, 2014.

Horion, S., Prishchepov, A. V., Verbesselt, J., Beurs, K., Tagesson, T., and Fensholt, R.: Revealing turning points in ecosystem functioning over the Northern Eurasian agricultural frontier, Glob. Change Biol., 22, 2801-2817, https://doi.org/10.1111/gcb.13267, 2016.

Ivits, E., Horion, S., Fensholt, R., and Cherlet, M.: Global Ecosystem Response Types Derived from the Standardized Precipitation Evapotranspiration Index and FPAR3g Series, Remote Sensing, 6, 4266-4288, https://doi.org/10.3390/rs6054266, 2014.

Jacob, L., Vert, J.-p., and Bach, F. R.: Clustered multi-task learning: A convex formulation, Adv. Neur. In., 745-752, 2009.

Köppen, W.: Das Geographische System der Klimate, Handbuch der klimatologie, Band I, Teil C, 1936.

Kottek, M., Grieser, J., Beck, C., Rudolf, B., and Rubel, F.: World map of the Köppen-Geiger climate classification updated, Meteorol. Z., 15, 259-263, 2006.

Li, W., MacBean, N., Ciais, P., Defourny, P., Lamarche, C., Bontemps, S., Houghton, R. A., and Peng, S.: Gross and net land cover changes in the main plant functional types derived from the annual ESA CCI land cover maps (1992-2015), Earth Syst. Sci. Data, 10, 219-234, https://doi.org/10.5194/essd-10-219-2018, 2018.

Liu, L., Zhang, Y., Wu, S., Li, S., and Qin, D.: Water memory effects and their impacts on global vegetation productivity and resilience, Scientific reports, 8, 2962, https://doi.org/10.1038/s41598-018-21339-4, 2018.

Liu, Y. Y., de Jeu, R. A. M., McCabe, M. F., Evans, J. P., and van Dijk, A. I. J. M.: Global long-term passive microwave satellitebased retrievals of vegetation optical depth, Geophys. Res. Lett., 38, L18402, https://doi.org/10.1029/2011GL048684, 2011. 
Loveland, T. and Belward, A.: The IGBP-DIS global $1 \mathrm{~km}$ land cover data set, DISCover: first results, Int. J. Remote Sens., 18, 3289-3295, 1997.

Loveland, T. R., Reed, B. C., Brown, J. F., Ohlen, D. O., Zhu, Z., Yang, L., and Merchant, J. W.: Development of a global land cover characteristics database and IGBP DISCover from $1 \mathrm{~km}$ AVHRR data, Int. J. Remote Sens., 21, 1303-1330, 2000.

Lund, R. and Li, B.: Revisiting climate region definitions via clustering, J. Climate, 22, 1787-1800, 2009.

Mahlstein, I., Daniel, J. S., and Solomon, S.: Pace of shifts in climate regions increases with global temperature, Nat. Clim. Change, 3, 739-743, 2013.

McQuade, S. and Monteleoni, C.: MRF-Based Spatial Expert Tracking of the Multi-Model Ensemble, in: The Third International Workshop on Climate Informatics National Center for Atmospheric Research, Boulder, Colorado, 26-27 September 2013, 3 pp., 2013.

Mekhalfi, M. L., Melgani, F., Bazi, Y., and Alajlan, N.: Landuse classification with compressive sensing multifeature fusion, IEEE Geosci. Remote S., 12, 2155-2159, 2015.

Metzger, M. J., Bunce, R. G. H., Jongman, R. H. G., Sayre, R., Trabucco, A., and Zomer, R.: A high-resolution bioclimate map of the world: a unifying framework for global biodiversity research and monitoring, Global Ecol. Biogeogr., 22, 630-638, https://doi.org/10.1111/geb.12022, 2012.

Nemani, R. R., Keeling, C. D., Hashimoto, H., Jolly, W. M., Piper, S. C., Tucker, C. J., Myneni, R. B., and Running, S. W.: Climatedriven increases in global terrestrial net primary production from 1982 to 1999 , Science, 300, 1560-1563, 2003.

Netzel, P. and Stepinski, T.: On using a clustering approach for global climate classification, J. Climate, 29, 3387-3401, 2016.

Netzel, P. and Stepinski, T. F.: World Climate Search and Classification Using a Dynamic Time Warping Similarity Function, in: Advances in Geocomputation, edited by: Griffith, D., Chun, Y., and Dean, D., Advances in Geographic Information Science, Springer, Cham, 181-195, https://doi.org/10.1007/978-3319-22786-3_17, 2017.

Ng, A. Y., Jordan, M. I., and Weiss, Y.: On spectral clustering: Analysis and an algorithm, in: Advances in Neural Information Processing systems, Vancouver, British Columbia, Canada 3-8 December 2001, edited by: Dietterich, T. G., Becker, S., and Ghahramani, Z., MIT Press, 849-856, 2001.

Papagiannopoulou, C., Miralles, D. G., Decubber, S., Demuzere, M., Verhoest, N. E. C., Dorigo, W. A., and Waegeman, W.: A non-linear Granger-causality framework to investigate climatevegetation dynamics, Geosci. Model Dev., 10, 1945-1960, https://doi.org/10.5194/gmd-10-1945-2017, 2017a.

Papagiannopoulou, C., Miralles, D. G., Dorigo, W. A., Verhoest, N. E. C., Depoorter, M., and Waegeman, W.: Vegetation anomalies caused by antecedent precipitation in most of the world, Environ. Res. Lett., 12, 074016, https://doi.org/10.1088/17489326/aa7145, 2017b.

Papagiannopoulou, C., Miralles, D. G., Demuzere, M., Verhoest, N. E. C., and Waegeman, W.: Hydro-Climatic biomes: a multitask learning approach, https://doi.org/10.5281/zenodo.1241047, last access: 20 September 2018.

Pedregosa, F., Varoquaux, G., Gramfort, A., Michel, V., Thirion, B., Grisel, O., Blondel, M., Prettenhofer, P., Weiss, R., Dubourg, V., Vanderplas, J., Passos, A., Cournapeau, D., Brucher, M., Per- rot, M., and Duchesnay, E.: Scikit-learn: Machine Learning in Python, J. Mach. Learn. Res., 12, 2825-2830, 2011.

Peel, M. C., Finlayson, B. L., and McMahon, T. A.: Updated world map of the Köppen-Geiger climate classification, Hydrol. Earth Syst. Sci., 11, 1633-1644, https://doi.org/10.5194/hess-11-16332007, 2007.

Poulter, B., MacBean, N., Hartley, A., Khlystova, I., Arino, O., Betts, R., Bontemps, S., Boettcher, M., Brockmann, C., Defourny, P., Hagemann, S., Herold, M., Kirches, G., Lamarche, C., Lederer, D., Ottlé, C., Peters, M., and Peylin, P.: Plant functional type classification for earth system models: results from the $\mathrm{Eu}-$ ropean Space Agency's Land Cover Climate Change Initiative, Geosci. Model Dev., 8, 2315-2328, https://doi.org/10.5194/gmd8-2315-2015, 2015.

Propastin, P., Kappas, M., and Erasmi, S.: Application of Geographically Weighted Regression to Investigate the Impact of Scale on Prediction Uncertainty by Modelling Relationship between Vegetation and Climate, International Journal of Spatial Data Infrastructures Research, 3, 73-94, 2008.

Scott, G. J., England, M. R., Starms, W. A., Marcum, R. A., and Davis, C. H.: Training deep convolutional neural networks for land-cover classification of high-resolution imagery, IEEE Geosci. Remote S., 14, 549-553, 2017.

Seddon, A. W., Macias-Fauria, M., Long, P. R., Benz, D., and Willis, K. J.: Sensitivity of global terrestrial ecosystems to climate variability, Nature, 531, 229-232, 2016.

Spinoni, J., Vogt, J., Naumann, G., Carrao, H., and Barbosa, P.: Towards identifying areas at climatological risk of desertification using the Köppen-Geiger classification and FAO aridity index, Int. J. Climatol., 35, 2210-2222, 2015.

Subbian, K. and Banerjee, A.: Climate multi-model regression using spatial smoothing, in: Proceedings of the 2013 SIAM International Conference on Data Mining, SIAM, 324-332, https://doi.org/10.1137/1.9781611972832.36, 2013.

Thornthwaite, C. W.: Problems in the classification of climates, Geogr. Rev., 33, 233-255, 1943.

Trewartha, G. and Horn, L.: An Introduction to Climate, McGrawHill, New York, 416 pp., 1980.

Tucker, C. J., Pinzon, J. E., Brown, M. E., Slayback, D. A., Pak, E. W., Mahoney, R., Vermote, E. F., and El Saleous, N.: An extended AVHRR 8-km NDVI dataset compatible with MODIS and SPOT vegetation NDVI data, Int. J. Remote Sens., 26, 44854498, 2005.

Wang, F., Wang, X., and Li, T.: Semi-supervised multi-task learning with task regularizations, in: 9th IEEE International Conference on Data Mining (ICDM'09), Miami, FL, USA, 6-9 December 2009, IEEE, 562-568, https://doi.org/10.1109/ICDM.2009.66, 2009 .

Ward, J. H.: Hierarchical Grouping to Optimize an Objective Function, J. Am. Stat. Assoc., 58, 236-244, https://doi.org/10.1080/01621459.1963.10500845, 1963.

$\mathrm{Xu}$, D. and Tian, Y.: A Comprehensive Survey of Clustering Algorithms, Annals of Data Science, 2, 165-193, https://doi.org/10.1007/s40745-015-0040-1, 2015.

$\mathrm{Xu}$, J., Tan, P.-N., Luo, L., and Zhou, J.: Gspartan: a geospatiotemporal multi-task learning framework for multi-location prediction, in: SIAM International Conference on Data Mining, Miami, Florida, USA, 5-7 May 2016, SIAM, 657-665, https://doi.org/10.1137/1.9781611974348.74, 2016. 
Xu, X., Li, W., Ran, Q., Du, Q., Gao, L., and Zhang, B.: Multisource Remote Sensing Data Classification Based on Convolutional Neural Network, IEEE T. Geosci. Remote, 56, 937-949, https://doi.org/10.1109/TGRS.2017.2756851, 2018.

Xue, Y., Liao, X., Carin, L., and Krishnapuram, B.: Multi-task learning for classification with dirichlet process priors, J. Mach. Learn. Res., 8, 35-63, 2007.

Zhang, D. and Shen, D.: Multi-modal multi-task learning for joint prediction of multiple regression and classification variables in Alzheimer's disease, Neuroimage, 59, 895-907, 2012.

Zhang, X. and Yan, X.: Spatiotemporal change in geographical distribution of global climate types in the context of climate warming, Clim. Dynam., 43, 595-605, 2014a.

Zhang, X. and Yan, X.: Temporal change of climate zones in China in the context of climate warming, Theor. Appl. Climatol., 115, $167-175,2014 b$.
Zhang, Z., Luo, P., Loy, C. C., and Tang, X.: Facial landmark detection by deep multi-task learning, in: European Conference on Computer Vision, Zurich, 6-12 September 2014, Springer, 94 $108,2014$.

Zhao, Z., Gao, J., Wang, Y., Liu, J., and Li, S.: Exploring spatially variable relationships between NDVI and climatic factors in a transition zone using geographically weighted regression, Theor. Appl. Climatol., 120, 507-519, 2015.

Zhou, J., Chen, J., and Ye, J.: Clustered multi-task learning via alternating structure optimization, Adv. Neur. In., 2011, 702-710, 2011.

Zscheischler, J., Mahecha, M. D., and Harmeling, S.: Climate classifications: the value of unsupervised clustering, Procedia Comput. Sci., 9, 897-906, 2012. 